\title{
INCENTIVO AO ALEITAMENTO MATERNO EM UNIDADES BÁSICAS DE SAÚDE DE SANTA MARIA-RS
}

Ruth M aurer da Silva', Hedionéia M aria Foletto Pivetta², Eilamaria L ibardoni Vieira? A mara Lúcia H olanda Tavares B attistel ${ }^{4}$, M ichelle Gracioli ${ }^{5}$, R osiana Cordeiro Righi ${ }^{6}$

RESUM 0: 0 ato de amamentar até os seis meses de vida é um método eficiente de atender não só os aspectos nutricionais, imunológicos, psicológicos ou o desenvolvimento do bebê, mas é igualmente benéfico para as mães, pois estimula o vínculo mãe-filho. 0 trabalho obj etivou incentivar 0 aleitamento materno nas U nidades B ásicas de Saúde (UBS) da R egião Oeste de Santa M aria-RS, com encontros semanais em grupos de gestantes, seguindo as instruções recomendadas pela I niciativa U nidade B ásicaA miga da A mamentação (IUBA A M ), tendo como fundamento a promoção, proteção e apoio ao al eitamento materno. Os professores e acadêmicos da área das Ciências da Saúde dos Cursos de Enfermagem, Terapia Ocupacional, Nutrição eFisioterapia do Centro U niversitário Franciscano (UNIFRA), desenvolveram um projeto em parceria com os profissionais integrantes da Secretaria de Saúde do M unicípio com a finalidade de diminuir o desmame precoce e tornar 0 aleitamento materno uma prática contínua.

DESCRITORES: A leitamento materno; Equipe multiprofissional; Promoção da saúde.

\section{ENCOURAGING BREAST-FEEDING IN PRIMARY CARE HEALTH UNITS IN SANTA MARIA-RS}

ABSTRACT: The act of breastfeeding until six months old, is an efficient method not only to meet nutritionist, immunologist, psychological aspects or babies' development, but also it is equally beneficial to mothers, strengthening mother/child's bond. The study aimed to stimulate breastfeeding in Primary $\mathrm{C}$ are $\mathrm{H}$ eal th U nits in the western region of Santa M aria-R S/B razil, through weekly meetings with groups of pregnant women, following the instructions recommended by the B reastfeeding- Friendly Primary Care U nit Initiative, founded on breastfeeding promotion, protection and support. Professors and academics of the Health A rea, that is, from Nursing, Occupational Therapy, Nutrition and Physical Therapy from Centro Universitário Franciscano (U NIFRA), had developed a project, in partnership with professionals from the Municipal Health Secretary, objectifying to decrease early weaning and make breastfeeding an ongoing practice.

DE SCRIPTORS: Breastfeeding; M ultiprofissional team; Promotion of the health.

\section{INCENTIVO AL AM AMANTAMIENTO MATERNAL EN LAS UNIDADES BÁSICAS DE LA SALUD DE SANTA MARIA-RS}

RESU M EN : EI hecho de amamantar hasta los seis meses de vida es un método eficiente no sólo para llevar cuidado del nutricionista, de los inmunologistas, de los aspectos psicologicos o del desarrollo del bebé, pero es igualmente beneficioso para las madres. EI trabajo tuvo el objetivo de estimular el amamantamiento en las U nidades B ásicas de Salud (UBS) de la región al oeste de Santa M aria - RS, con encuentros semanal es en grupos de gestante según las instrucciones de la I niciativa U nidad B ásicaA miga del A mamantamiento (IU BAA M ), que tiene como fundamento la promoción, proteción y apoyo al amamantamiento materno. Los profesores y académicos del área de las Ciencias de la Sal ud de los Cursos de Enfermería, Terapia Ocupacional, N utrición y Fisioterapia del Centro U niversitario Franciscano (UNIFRA) desarrollaron un proyecto en sociedad con los profesionales de la Secretaría de Salud del M unicipio con la finalidad de disminuir el destete precoce y volver el amamantamiento materno una práctica continua. DESCRIPTORE S: A mamantamiento; Equipo multipropfesional; Promoción de la salud.

\footnotetext{
${ }^{1}$ M estre em Ciência e Tecnologia Farmacêuticas pela Universidade Federal de Santa M aria - UFSM. Docente do Curso de Nutrição da Área de Ciências da Saúde do Centro U niversitário Franciscano - UNIFRA.M embro do Grupo Interdisciplinar de Pesquisas em Saúde - GIPES.

2 M estranda. Fisioterapeuta. Docente do Curso de Fisioterapia do UNIFRA.

${ }^{3}$ M estre em Ciência dos A limentos pela Universidade Federal de Santa Catarina - UFSC. Nutricionista. Docente do Curso de Nutrição da Á rea de Ciências da Saúde do UNIFRA.

${ }^{4} \mathrm{M}$ estre em Educação pela UFSM . Coordenadora e docente do Curso de Terapia Ocupacional do UNIFRA.

${ }^{5}$ M estre em Enfermagem pela UFSC. Docente do Curso de Enfermagem da Á rea de Ciências da Saúde do U NIFRA. M embro do GIPES.

${ }^{6}$ A cadêmica do Curso de Nutrição do UN IFRA. Colaboradora do Projeto.
}

$R$ dos A ndradas, 1614 - 97010-032 - Santa M aria-RS Recebido em: 04/09/06

E-mail: reitoria@unifra.br 


\section{INTRODUÇÃO}

A importância do aleitamento materno exclusivo até os seis meses é um fato amplamente reconhecido pelos profissionais de saúde, porquanto, o leite materno representa o melhor alimento para o bebê. Por isso a Organização M undial da Saúde (OM S), as instituições e órgãos não-governamentais estudam e divulgam o assunto e são unânimes em sua orientação: apoiar a prática do aleitamento materno exclusivo até seis meses e complementado até dois anos de idade visando mel horar a qual idade de vida e reduzir a desnutrição, a mortalidade infantil e as doenças crônicas não transmissíveis na idade adulta.

Estudos têm mostrado redução no tempo de amamentação exclusiva, e tal procedimento é justificado por vários fatores, entre eles, a falta de preparação das mul heres para a amamentação durante o pré-natal.

Considerando ser o desmame precoce um problema de Saúde Pública, professores e acadêmicos da Á rea das Ciências da Saúde, dos Cursos de Enfermagem, Terapia Ocupacional, Nutrição e Técnico de Enfermagem do Centro Universitário Franciscano (U NIFRA), desenvolveram um projeto em U nidades B ásicas de Saúde do M unicípio de Santa $M$ aria-RS, em parceria com profissionais integrantes da Secretaria de Saúde do M unicípio: enfermeiros, médicos e agentes de saúde, com o objetivo de incentivar 0 aleitamento materno seguindo as instruções recomendadas pela Iniciativa U nidade $B$ ásica A miga daA mamentação (IU B A A M ), que tem como fundamento a promoção, proteção e apoio ao al eitamento materno por meio da mobilização das Unidades Básicas de Saúde para a adoção dos "Dez Passos para o Sucesso da A mamentação" da IUBAAM.

\section{REVISÃO BIBLIOGRÁFICA}

A gestação é um fenômeno fisiológico que acarreta uma série de modificações no organismo da mãe, com a finalidade de garantir o crescimento fetal, proteger o organismo materno e ainda, possibilitar a recuperação da puérpera e a nutrição de recém-nascido(1).

O corpo começa a ser preparado para a amamentação já na gravidez, por isso é importante aval iar as mamas nas consul tas do pré-natal e examinar também as condições dos mamilos ${ }^{(2)}$. A gestação compreende um período de grande vulnerabilidade para a mãe, em razão das várias transformações em seu corpo, e para o feto, devido ao seu crescimento e desenvolvimento(1).

A desnutrição protéico-calórica e as deficiências deferro, iodo, vitaminaA efolato, principais problemas nutricionais na gestação, deverão ser avaliados com base no impacto que exercem não somente no organismo da gestante, como também no do feto/recémnascido. Se a gestante apresentar menores reservas nutricionais, há um maior risco do feto e do recémnascido apresentarem déficit do desenvolvimento neurocognitivo, mal formações congênitas, prematuridade, levando ao nascimento de crianças pequenas para a idade gestacional ${ }^{(1)}$. A gestante deve ter uma dieta variada, mas deve evitar alimentos gordurosos e pesados, café e açúcar em excesso e não consumir álcool e nem fumar cigarro(3).

0 peso recomendado durante a gravidez varia segundo o estado nutricional pré-gestação. Às mulheres que se encontram com baixo peso é permitido um ganho ponderal de peso, já àquelas anteriormente obesas é recomendado um pequeno ganho de peso, porém nunca inferior a seis quilogramas ${ }^{(4)}$. U ma boa nutrição, durante a gravidez ajuda na formação de bebês saudáveis e contribui para o sucesso da amamentação( ${ }^{(5)}$.

Como na gestação, a lactação, é um momento especial na vida da mulher, e como tal, precisa de cuidados especiais ${ }^{(6)}$. A pós o parto, as mulheres devem ser estimuladas a manter um bom estado nutricional para que elas mesmas ajudem a promover uma energia necessária para cuidar do bebê e logo iniciar a amamentação(2,7).

0 leite materno é indicado como alimento ideal para a criança nos seus primeiros meses de vida, devido às suas propriedades nutricionais e antiinfecciosas, além das vantagens psicossociais da prática do al eitamento para a mãe e seu filho ${ }^{(8)}$.

A lactação - produção e secreção de leite - é um processo natural ${ }^{(4)}$. 0 primeiro leite produzido pela mãe secretado até três dias após o parto, é denominado colostro e apresenta características específicas, adequadas às necessidades do recém-nascido, sendo a primeira imunização para proteger a criança contra a maior parte das bactérias e vírus, desempenhando assim, um papel mais protetor do que nutricional. Por isso, ele tem menor teor de gordura e de calorias e maior quantidade de fatores imunológicos ${ }^{(9)}$.

0 colostro é também rico em fatores de crescimento que estimulam o intestino maduro da criança 
a se desenvolver. 0 fator de crescimento prepara 0 intestino para diferenciar e absorver o leite maduro e impede a absorção de proteínas não digeridas. Se a criança recebe leite de vaca ou de outro animal antes de receber 0 colostro, estes al imentos podem lesar 0 intestino e causar al ergias ${ }^{(10)}$.

0 leite materno é produzido para satisfazer as necessidades de nutrientes do lactente humano. Seu carboidrato é a lactose, sua proteína é especial mente digerível e utilizável para sustentar o crescimento tecidual. $E$ sua gordura fornece uma porção generosa de ácido graxo essencial ômega-6 ácido linoléico e seus produtos ${ }^{(11)}$.

A lém disso, a mãe que consome al imentos ricos em ácidos graxos essenciais ômega-3 passa esse nutrientes benéficos para o seu filho por meio do seu leite, proporcionando esse importante substrato para o desenvolvimento do sistema nervoso e da retina do lactente ${ }^{(9)}$.

A ssim, com uma amamentação adequada, a criança consegue manter os níveis ideais de concentração de vitamina $A$, sendo o leite materno em geral, a maior fonte dessa vitamina nos primeiros 24 meses de vida ${ }^{(5)}$. A vitamina A é importante para 0 bom desenvolvimento dos olhos, ajuda a reduzir a gravidade de infecções comuns em crianças e auxilia no desenvolvimento e crescimento delas ${ }^{(12)}$.

A s vantagens do aleitamento materno: é nutricional mente completo e superior a qual quer outro tipo de leite para o bebê, é sempre fresco, na temperatura ideal, não precisa ser misturado a nada e é gratuito, já que a alimentação artificial pode consumir de $20 \%$ a $90 \%$ da renda familiar ${ }^{(5)}$.

0 benefício da amamentação vale por toda a vida. Crianças que mamam têm menos risco de sofrer de doenças respiratórias, infecções urinárias ou diarréias, problemas que podem levar a internações e até à morte. 0 bebê amamentado corretamente terá menos chance de desenvolver diabetes, hipertensão e doenças cardiovasculares ${ }^{(13)}$.

São inúmeras as vantagens da amamentação, especialmente nos primeiros meses de vida, porquanto ela garante em muitos casos, a sobrevivência das crianças e particularmente daqueles em condições desfavoráveis ou que nasceram com baixo peso. Do ponto de vista nutricional a complementação al imentar precoce é desvantajosa para a criança, pois além de reduzir a duração do al eitamento materno prejudica a absorção de nutrientes importantes ${ }^{(14)}$.

Compreende-se por aleitamento materno exclusivo quando a criança recebe somente leite materno, diretamente da mama ou extraído, e nenhum outro líquido ou sólido com exceção de remédios. Ele garante, em muitos casos, a sobrevivência das crianças principalmente daquel as em condições desfavoráveis ou que nascem de baixo peso. Q uanto mais a criança mamar no peito maior sua proteção(15).

0 leite materno traz benefícios não só ao bebê, mas também para as mulheres, famílias, comunidades e meio ambiente. A mulher que amamenta já na maternidade corre menos risco de ter hemorragia no pós-parto e anemia. 0 al eitamento materno também ajuda no espaçamento entre uma gestação e outra, dando à mul her mais tempo para recuperar-se do parto e cuidar do bebê, protege contra o câncer de mama e de ovário, permite-lhe recuperar mais facilmente 0 peso que tinha antes da gravidez, facilita remineralização prevenindo fraturas vertebrais e do fêmur no período da menopausa ${ }^{(3,16)}$.

Os bebês amamentados serão mais saudáveis e inteligentes e desta forma as comunidades também serão beneficiadas. Vivemos em um mundo cada vez mais poluído, e o aleitamento materno evita desperdício de recursos e agressões ao meio ambiente, pois cada mãe que amamenta reduz o problema de contaminação e excesso de lix $0^{(5,17) \text {. }}$

No B rasil, tem-se procurado resgatar a prática do al eitamento materno através de várias propostas como o Programa Nacional de Incentivo do A leitamento $M$ aterno, tanto nas unidades básicas de saúde, estas procurando seguir as normas da IU BAAM , como nas iniciativas do Hospital A migo da Criança, destinado a estimular hospitais e maternidades a adotarem os dez passos para o sucesso do al eitamento materno ${ }^{(8)}$.

A OMS recomenda 0 aleitamento materno exclusivo nos primeiros seis meses de vida(18). No entanto essa não é uma questão fechada e há quem defenda amamentação exclusiva além desse período ${ }^{(15)}$.

A própria OM S orienta que a amamentação pode ser complementada até 2 anos ou mais. No segundo ano de vida, o leite materno continua sendo uma importante fonte de nutrientes, além de continuar conferindo proteção contra doenças infecciosas ${ }^{(17)}$. Em al gumas populações, el e contribui com um a dois terços da energia ingerida no final do primeiro ano e continua sendo uma importante fonte de gordura, vitamina A, cál cio e riboflavina no segundo ano de vida(15).

Certas mães sentem-se felizes em amamentar, outras, ao contrário, sofrem influências negativas de pessoas mal orientadas e preferem dar a mamadeira, 
parando rapidamente o processo de amamentação ou oferecem a mamadeira como complemento ${ }^{(3)}$.

A possibilidade de que as mulheres que trabalham mantenham a amamentação é um dos desafios que atualmente os profissionais de saúde interessados na promoção do al eitamento estão enfrentando. A situação de cada mulher deve ser analisada de forma individual e antecipada, pelo menos um mês antes dela voltar ao trabalho. A legislação brasileira prevê uma licença após o parto de até quatro meses e dois intervalos de meia hora cada, durante as horas de trabal ho (ou saída uma hora antes) para que a mãe possa amamentar o seu filho até no mínimo seis meses de idade ${ }^{(17)}$.

A ntes de retornar ao trabal ho a mãe pode praticar a ordenha do leite e congelá-lo para usar mais tarde. Deve-se iniciar o estoque vinte dias antes de retornar ao trabal ho. A pós o retorno do trabal ho, amamentar com freqüência quando estiver em casa, evitando as mamadeiras, oferecer 0 al eitamento com copo ou colher, durante as horas de trabal ho esvaziar as mamas de forma manual e guardar o leite na geladeira ${ }^{(17)}$.

Para prevenir fissuras, é indicado ainda na gestação expor os mamilos ao sol por quinze minutos no horário antes das dez horas ou após as dezesseis horas $^{(2)}$. 0 estresse, a ansiedade e a dor constituem problemas que podem interferir na produção de leite ${ }^{(3)}$.

Todo o processo biológico e de desenvolvimento do lactente pode ser influenciado pela prática alimentar nos primeiros seis meses de vida. Essa prática vai prevenir as alergias alimentares, a desnutrição, a obesidade, a anemia, as deficiências nutricionais, 0 diabetes melito e a constipação ${ }^{(9)}$.

Depois de seis meses o lactente deve receber alimentos em formas de papas que Ihe forneçam calorias, proteínas e fazer o possível para que os alimentos contenham ferro de origem animal. Dessa forma, a introdução de alimentos complementares pode parecer interessante para a criança exclusivamente amamentada e que apresenta uma desaceleração do ganho de peso, por outro lado, essa introdução, com freqüência, representa maior risco de infecções e, em situações de pobreza extrema, até mesmo maior risco de morte ${ }^{(15)}$.

0 leite materno, apesar de sua baixa quantidade de ferro, previne a anemia nos seis primeiros anos de vida da criança, pois a biodisponibilidade permite a absorção de $50 \%$ do ferro presente, enquanto a absorção do ferro do leite de vaca, que tem quantidades semelhantes, é de apenas $10 \%{ }^{(9)}$.
O cuidar-orientar-ouvir humanizado do profissional de saúde durante o ciclo grávidopuerperal fortalece a estabilidade, harmonia, entrosamento entre a gestante e a equipe de saúde, propiciando integração, qualidade do cuidado prestado e reconhecimento entre as partes, pois a gestante somente poderá expor seus temores, dúvidas, angústias e dificuldades se instituir o vínculo de suporte confiável, em meio a tantas modificações e sensações novas ${ }^{(19)}$.

Portanto, os grupos de gestantes trazem aspectos terapêuticos e of erecem suporte às gestantes, uma vez que "um grupo pode ajudar pessoas durante período de ajustamentos a mudanças, no tratamento de crises ou ainda na manutenção ou adaptação a novas situações"(20:20). A ssim, os grupos devem oferecer condições para que apreendam, aceitem e pratiquem conhecimentos e atitudes relativos à gestação, ao parto, ao puerpério e ao recém-nascido e também à prática de exercícios corporais.

Para que a gestante esteja preparada para ser mãe é importante que tenha conhecimentos sobre 0 processo de gestação, parto e pós-parto, al eitamento materno, rotinas médicas, hospitalares, considerando que o conhecimento gera poder, assim como poder gera conhecimento. Dessa forma, a gestante e 0 companheiro/familiar que expressam seu sentimento acerca deste período tornam-se menos ansiosos e 0 medo frente ao desconhecido tende a diminuir. N esta perspectiva, atual mente, os grupos de gestantes vêm sendo coordenados por equipes interdisciplinares, a fim de prestar apoio de forma completa e satisfatória para a gestante ${ }^{(21)}$.

\section{METODOLOGIA}

\section{População de estudo}

0 projeto foi desenvolvido em quarenta e oito grupos de gestantes, de seis Unidades Básicas de Saúde da R egião O este de Santa M aria-RS, no período de março a dezembro de 2005, totalizando 282 gestantes participantes nas atividades desenvolvidas.

\section{Delineamento de estudo}

A s gestantes participaram dos grupos por livre demanda. A divulgação foi feita na unidade de saúde por meio de cartazes, convite escrito e pessoal. 
Por esta pesquisa envolver seres humanos segundo a Resolução do Consel ho N acional de Saúde - CNS 196/96, foi utilizado do Termo de Consentimento Livre Esclarecido - TCLE.

\section{Procedimentos}

Foram realizados encontros semanais, quinzenais ou mensais conforme a demanda de cada unidade, coordenada pela equipe de Saúde das Unidades Básicas de Saúde (UBS) e pela Equipe de Saúde do Centro Universitário Franciscano, anteriormente mencionados.

Para determinar as atividades a serem desenvolvidas foi realizado um encontro de diagnóstico educativo em cada UBS e a partir das necessidades de cada grupo foi proposto o cronograma de atividades, procurando seguir as instruções recomendadas pela Iniciativa Básica A miga da A mamentação.

Foram desenvolvidas dinâmicas educativas interdisciplinares sobre as modificações e os desconfortos da gestação, al eitamento materno, alimentação na gestação e lactação, alimentação no primeiro ano de vida, tabus e crendices populares, cuidados com o corpo e com as mamas e preparação para o parto.

As técnicas utilizadas foram: conversas expositivas e com auxílio de cartazes e folder educativos a partir de suas dúvidas e curiosidades; oficinas com distribuição de folder e folhetos educativos; entrevistas, cartazes e jogos educativos.

\section{DISCUSSÃO E RESULTADOS}

A s experiências demonstram que atividades de educação em saúde devem ser planejadas de acordo com os aspectos sociais, econômicos, psicológicos e culturais do grupo e que o papel profissional de saúde como educador é antes de tudo contribuir para que as gestantes conquistem condições de vida mais humanas, favoráveis ao desenvolvimento pleno das potencialidades físicas, intelectuais, emocionais e espirituais.

Os diferentes tipos de recursos utilizados proporcionaram a realização de atividades lúdicas com os grupos, respeitando as condições sócio-econômicas e culturais das participantes do grupo. Segundo relatos das gestantes, a utilização de estratégias de educação em saúde que associam a af etividade, a ludicidade ea criatividade têm maior efetividade, o que contribui para 0 aprendizado.

De acordo com a avaliação dos profissionais das UBS a utilização das instruções recomendadas pela Iniciativa Unidade Básica Amiga da A mamentação (IUBAAM) associadas com metodologias adequadas contribuiu para mel horia da qualidade de vida das gestantes e incentivo ao al eitamento materno. 0 vínculo criado foi destacado pelas gestantes como fator de sucesso do projeto.

Observamos maior participação das gestantes primíparas e percebemos que tanto as visitas às unidades de saúde como à comunidade, quanto à conversa expositiva foram meios eficientes de orientar e levar informações à comunidade.

A lém da integração entre os participantes, houve momentos de descontração, diversidade de temas abordados, criou-se principalmente vínculos entre a equipe organizadora e comunidade. Esta iniciativa del ineia importante papel de suporte que as U nidades B ásicas de Saúde podem desempenhar a fim de tornar 0 aleitamento materno uma prática universal, contribuindo significativamente para a saúde e bem estar dos bebês e suas mães.

Este método de ensino-aprendizagem criou oportunidades de conhecimento nas áreas afetivas, cognitivas e psico-motora, favorecendo 0 desenvolvimento da dimensão crítico-social. Nesta dimensão inseriu-se a ação assistencial em saúde nas quais se inclui a promoção, proteção e apoio à amamentação.

Durante 0 desenvolvimento das atividades, as participantes mostraram-se interessadas, e pode-se perceber que a maioria gostaria de amamentar, tendo consciência das vantagens que isso irá trazer para 0 bebê e para elas.

\section{CONSIDERAÇÕES FINAIS}

A gestação é um momento peculiar para a mulher, na qual se evidenciam várias transformações, tanto físicas como psicológicas, exigindo adaptações que não decorrem só de aprendizagem cognitiva, mas de elaboração da vivência, sendo fundamental a interação da família e/ou apoio de grupos.

Nesse sentido, professores e acadêmicos da Área das Ciências da Saúde dos Cursos de Enfermagem, Terapia O cupacional, Nutrição e Fisioterapia do Centro Universitário Franciscano (UNIFRA), através de encontros com gestantes, 
contribuíram na vida destas gestantes, amenizando suas angústias bem como informando sobre a prática e a importância do aleitamento materno.

0 engajamento no trabal ho grupal despertou 0 interesse das gestantes pelo al eitamento materno, que ao ser difundido nesses grupos possibilitou a troca de vivências e de dificuldades, mostrando que essas atividades favorecem a interação frente a uma nova experiência, o aleitamento. A participação ativa e dinâmica tanto dos profissionais da saúde como das gestantes e familiares facilitou a discussão dos temas abordados durante os encontros, renovado no dia-adia, através de novas temáticas.

Percebeu-se que esse espaço de trocas de conhecimento serviu como elo de ligação entre teoria e prática, com isso ações de educação continuada neste âmbito servem como subsídio para que o incentivo ao aleitamento materno seja praticado.

\section{REFERÊNCIAS}

1. GoulartRM M, YazlleM EH, Rondó P, Vitolo M, Czeizela A. N ovas recomendações nutricionais para gestantes. $\mathrm{Rev}$ Nutr Pauta 2000; 8(45):11-17.

2. Ctenas $M L$, Vitolo M R. C rescendo com saúde- o guia de crescimento da criança. São Paulo: C2; 1999.

3. Battista E. A alimentação do bebê - a importância e as vantagens da a amamentação. Vida e Saúde 2003;2(65):12-15.

4. Bassoul E, B runo P, K ritz S. Nutrição e dietética. 2a ed. Rio deJ aneiro: Senac; 1998.

5. Esterik PV. A mamentação on line [acesso em 2003 A go 31]. Disponible em: http://www.al eitamento.org.br/ asseguranca.htm.

6. Elias M C. A mamentação [acesso 2003 J un 11]. Disponível em: http://www.clubedobebe.com.br

7. Peckenpaugh NJ, Poleman CM .. Nutrição, essência e dietoterapia. 7å ed. São Paulo: Roca; 1997.

8. Percegoni N, A raújo RMA, Silva MMS et al. Conhecimento sobre aleitamento materno de puérperas atendidas em dois hospitais de Viçosa, M inas Gerais. Rev Nutri 2002; 15(1):15-28.

9. Vitolo MR. Nutrição da gestação à adolescência. São Paulo: Reichmann eAffonso; 2003.

10. A ccioly E, Sauders C, Lacerda EM A. Nutrição em obstetrícia e pediatria. Rio de Janeiro: Cultura M édica; 1998.

11. Sizer F, Whitney E. N utrição: conceitos e controvérsias. 8ạ ed. São Paulo: M anole; 2003.

12. Carrasa $F R, M$ arcondes $E$. Nutrição clínica em pediatria. São Paulo: Savier; 1991.

13. Salviano S. 2004. Leite materno é saúde. Rev Nutri Pauta. 67(12). São Paulo: Núcleo Consultoria.

14. M ahan KL, Escott-Stump S. K rause - alimentos, nutrição dietoterapia. 10ª ed. São Paulo: R oca; 2002.

15. M inistério da Saúde(BR). Guia alimentar para crianças menores de dois anos. B rasília; 2002.

16. Lang S. A leitamento do lactente - cuidados especiais. São Paulo: Santos; 1999.

17. Giugliani, ERJ. 0 aleitamento materno na prática clínica. J Pediatr 2002; 76(03):238-52.

18. Oliveira MIC, Camacho LAB. Impacto das unidades básicas de saúde na duração do al eitamento materno exclusivo. Rev Bras Epidemiol 2002; 5(1):41-51.

19. M achado M V P, Zagonel IPS. 0 processo de cuidar da adolescente que vivencia a transição ao papel materno. Cogitare Enferm 2003; 2(8):26-33.

20. Wall ML. Tecnologias educativas: subsídios para a assistência de enfermagem a grupos. Goiânia: $A B$; 2001.

21. Pichon-RivièreE. 0 processo grupal. $6^{a}$ ed. São Paulo: $M$ artins F ontes, 2000.

Cogitare Enferm 2007 jan/mar; 12(1):95-100 